\title{
Herramientas GIS al servicio del análisis y la gestión de grandes áreas de actividad económica. El caso de la Comunitat Valenciana.
}

\section{Ana Isabel Escoms Martínez ${ }^{1}$, Adrián García Martínez², Carlos Esteve Aguado ${ }^{3}$, Juan Jaime Cano Hurtado ${ }^{4}$}

\footnotetext{
${ }^{13}$ Departamento de Urbanismo, ${ }^{24}$ Departamento de Ingeniería de la Construcción y de Proyectos de Ingeniería Civil. Universidad Politécnica de Valencia, Valencia, España

E-mail: 106349@ctav.es, ${ }^{2}$ adriangarma@gmail.com, 33carlosesteve3@gmail.com, ${ }^{3 j j}$ cano@cst.upv.es
}

\begin{abstract}
Resumen. En los polígonos industriales se desarrollan las actividades económicas que constituyen uno de los principales motores de la economía de la Comunitat Valenciana, ocupando una parte muy importante de nuestro territorio y, salvo en muy contados casos, se ha descuidado su calidad urbana, así como su integración medioambiental y paisajística. A esta circunstancia se añade la escasa inversión que se realiza en estas áreas para su mantenimiento y conservación. Todo ello, en el marco de una crisis económica sin precedentes, ha llevado a su desocupación, e incluso, a su obsolescencia.

La situación demanda actuaciones de renovación, regeneración, actualización, reordenación, especialización y un largo etcétera de mejoras.

Para establecer las estrategias de actuación, es necesario un análisis exhaustivo a nivel territorial, que establezca criterios adecuados para decidir el destino de las inversiones. Las actuaciones a nivel municipal/local se han demostrado ineficaces y han desembocado en una falta de criterios unificados de inversión. Es preciso abordar éstas sobre áreas conjuntas, en base a su localización o a su actividad económica, y desarrollar sinergias que aumentan su competitividad optimizando los recursos empleados.

Para una adecuada gestión de los datos en territorios de magnitud supramunicipal, es fundamental la utilización de herramientas basadas en Sistemas de Información Geográfica (GIS) que, además de manejar gran cantidad de datos, permiten un análisis exhaustivo en base a una realidad fisica. El artículo pretende establecer unos criterios básicos para la realización de este análisis a través del GIS, como herramienta de gestión del territorio.
\end{abstract}

Palabras clave: industria, GIS, análisis, regeneración, estrategia, territorio.

\section{Introducción. Sistemas de Información Geográfica.}

Aunque los Sistemas de Información Geográfica (SIG o GIS) empezaron siendo herramientas de la cartografía y de la geografía, ya tienen aplicación en cualquier disciplina. $\mathrm{Su}$ potencial es innegable, pero es tanto más útil en aquellas áreas de estudio donde más importancia tiene la localización de los datos que se manejan. En los últimos años, además, dada la facilidad de producir y obtener este tipo de datos, georreferenciados, han pasado a ser un elemento cotidiano.

La regulación en la Unión Europea tiene el objetivo de poner la información geográfica al alcance de todos los ciudadanos. La Directiva Inspire (Infraestructure for Spatial Information 


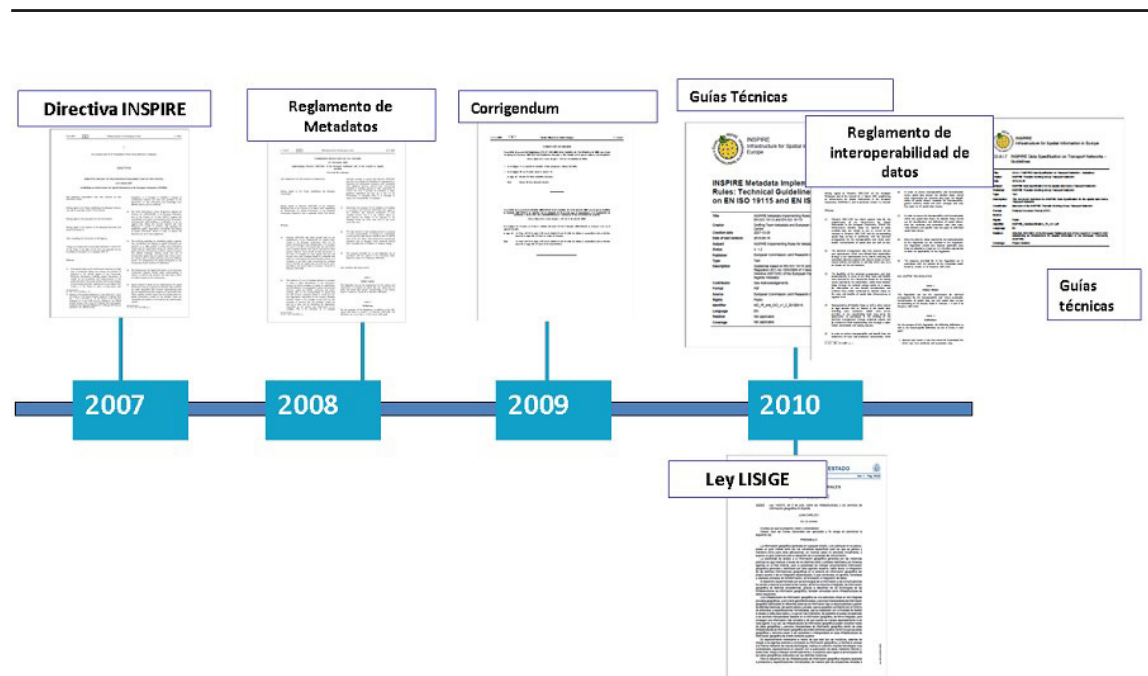

Fig. 1. Cronograma de la transposición de la Directiva INSPIRE en España. Fuente: Ministerio de Fomento.

in Europe) (Directiva 2007/2/CE) establece las reglas generales para la creación de una Infraestructura de Información Espacial en la Comunidad Europea a partir de las Infraestructuras de sus Estados miembros. La transposición de esta Directiva al ordenamiento jurídico español se ha desarrollado a través de la Ley 14/2010, de 5 de julio, sobre las infraestructuras y los servicios de información geográfica en España (LISIGE) (modificada por la Ley $2 / 2018$ de 23 de mayo) y es el Consejo Superior Geográfico el encargado del desarrollo de la Directiva Inspire en España.

ElRealDecreto 4/2010delEsquema Nacional de Interoperabilidad establece los criterios y recomendaciones de las Admnistraciones Públicas en este sentido y el Real Decreto 1545/2007 regula el Sistema Cartográfico Nacional. De esta forma se promueve la puesta en marcha de las Infraestructuras de Datos Espaciales (IDE) que facilita el acceso a la información geográfica y el uso de geoservicios de una manera regulada. Los datos geográficos y servicios proporcionados por las distintas Administraciones $u$ organismos del sector público integrados en la Infraestructura de Información Geográfica de España están disponibles a través del Geoportal de la Infraestructura de Datos Espaciales de España (IDEE) del Instituto Geográfico Nacional. La Comunitat Valenciana se encuentra integrada en este sistema en base al Convenio de Colaboración con el Instituto Geográfico Nacional del 1 de junio de 2010. La Infraestructura Valenciana de Datos Espaciales
(IDEV) integra la información en un mismo portal. El Institut Cartogràfic Valencià tiene atribuidas las funciones de coordinación de la Infraestructura Valenciana de Datos Espaciales (IDEV),

\section{Sistema Geodésico de Referencia y Sistema de Coordenadas}

Mediante el Real Decreto 1071/2007, de 27 de Julio de 2007, por el que se regula el Sistema Geodésico de Referencia oficial en España, ésta adopta como sistema de referencia geodésico global el ETRS89, sustituyendo al sistema geodésico de referencia regional ED50 sobre el que actualmente se referenciaba toda la cartografía oficial en el ámbito de la Península Ibérica y las Islas Baleares, y el sistema REGCAN95 en el ámbito de las Islas Canarias. Con la adopción del ETRS89 se obtiene una completa integración de la cartografía oficial española con los sistemas de navegación y la cartografía de otros países europeos.

El Sistema de Coordenadas Universal Transversal de Mercator (en inglés Universal Transverse Mercator, UTM) es un sistema de coordenadas basado en la proyección cartográfica transversa de Mercator, donde se proyecta la Tierra en un cilindro transversal al eje de la misma. Mediante este sistema la Tierra queda dividida en husos (espacios comprendidos entre dos meridianos). A excepción de pequeñas áreas localizadas al oeste del meridiano 0, la Comunitat Valenciana está englobada en el Huso 30. Todos los mapas 
que aparecen en este trabajo están referenciados al sistema geodésico ETRS89 y al Sistema de Coordenadas de Proyección UTM30.

\section{Análisis de las Áreas de Actividades Económicas (AAE)}

¿Cuáles son las situaciones en las que podemos encontrarnos un suelo que impliquen diferencias considerables a la hora de invertir en él o desarrollar una actuación? Para analizar el estado de los suelos destinados a las áreas de actividad económica (AAE), debemos conocer entre otros, su situación urbanística, y su situación de desarrollo. Para ello nos ayudaremos de los Sistemas de Información Geográfica. A grandes rasgos, podemos establecer las siguientes situaciones:

-Situación urbanística del suelo. Suelo clasificado / planeado / programado.

Es el suelo que, aunque tiene las características físicas de un suelo rural, está clasificado como urbanizable (de tipo terciario o industrial, que son las calificaciones apropiadas para las AAE), tiene o no, un planeamiento aprobado y está o no programado para desarrollarse. También puede darse el caso de suelos que, siendo urbanos o urbanizables, tienen la calificación de residencial.

-Situación de desarrollo del suelo. Urbanización/ Edificación

Otra característica que hemos de conocer en los suelos destinados a las AAE es el estado de la urbanización, es decir, si ésta está o no ejecutada. Con ello diferenciaremos los suelos que se encuentran clasificados o programados de los que, efectivamente, han sido urbanizados. En los que se encuentren urbanizados deberemos analizar qué grado de colmatación por la edificación poseen. Finalmente, en un futuro estadio, deberíamos conocer cuáles de esas edificaciones se encuentran operativos, es decir, contienen una actividad en el momento del análisis.

Situación urbanística del suelo.

Para conocer la situación urbanística del suelo destinado a las AAE, deberíamos recopilar todos los instrumentos de planeamiento urbanístico que tienen potestad para clasificar, calificar, planear o programar suelo. Esta información ya ha sido elaborada y es ofrecida por la Infraestructura Valenciana de Dades Espacials (IDEV). De estos datos obtendremos la siguiente información:

- Clasificación del Suelo.

Los instrumentos de planeamiento clasifican el suelo en urbano, urbanizable y no urbanizable. El suelo urbano lo constituyen los solares y los terrenos urbanizados, es decir, aquellos que cuentan con los servicios urbanísticos o sus colindantes, dado que con pequeñas actuaciones urbanísticas pasarían a formar parte de la trama urbana. El suelo urbanizable lo constituyen aquellos suelos de zonas de nuevo desarrollo y expansión, es decir, supone la mera aptitud de los terrenos para su urbanización, previa programación de los mismos. El suelo no urbanizable lo constituyen las zonas rurales.

- Zonificación del Suelo.

El territorio se zonifica en función de las características del territorio, de sus valores y de los usos a implantar, así que además de la clasificación del suelo, tenemos la calificación del suelo, es decir, si su uso dominante es residencial, terciario o industrial, para suelos urbanos o urbanizables, y si es un suelo común o protegido en caso de zonas rurales, así como su carácter principal, forestal, dominio público, etc. Además, de estos datos, obtenemos más información:

- Delimitación de ámbitos de planeamiento urbanístico diferenciado.

- Red de dotaciones públicas y privadas, y sus características.

\section{Situación de desarrollo del suelo}

Una vez conocida la situación urbanística del suelo, necesitamos conocer su efectivo desarrollo, para ello, tenemos diferentes herramientas:

Sistema de Información sobre Ocupación del Suelo de España (SIOSE).

El objetivo de este sistema, integrado dentro del Plan Nacional de Observación del Territorio (PNOT), es generar una base de datos de ocupación del suelo para toda España a escala de referencia 1:25.000, integrando la información disponible de las comunidades 
autónomas y la Administración General del Estado. El SIOSE se ha producido a nivel nacional a fecha de referencia del año 2005 (SIOSE 2005), con posteriores actualizaciones a fecha de referencia 2009 (SIOSE 2009) y 2011 (SIOSE 2011).

\section{CORINE Land Cover}

El 27 de junio de 1985, en virtud de una decisión del Consejo de Ministros de la Unión Europea (CE/338/85), se inició el programa Corine (coordinación de la información sobre el medio ambiente) en la Unión Europea. fue un proyecto prototipo que trabaja en muchos temas ambientales diferentes, "un proyecto experimental para la recopilación, la coordinación y la homogenización de la información sobre el estado del medio ambiente y los recursos naturales en la Comunidad"

Dentro de este programa se crea el proyecto CORINE Land Cover (CLC), que desde 1995 es responsabilidad de la Agencia Europea del Medio Ambiente. con el objetivo fundamental de obtener una base de datos europea de ocupación del suelo a escala 1:100.000, útil para el análisis territorial y la gestión de políticas europeas. Este programa es un auténtico inventario de la cobertura del suelo en 44 clases. En la actualidad este proyecto está incluido en el Land Core Monitoring System de GMES (Global Monitoring for
Environment and Security), una iniciativa de la Unión Europea (UE) para desarrollar su propia capacidad operativa de observación de la Tierra en Europa.

Se observa que el SIOSE tiene una escala de referencia menor, por lo que es más detallado. En este sistema, las coberturas que nos interesan son aquellas referidas a usos/ coberturas referidos a las AAE, es el caso de los Suelos Artificiales Compuestos Primarios/ Industriales y/o Terciarios. También algunas dotaciones como los puertos, aeropuertos, estaciones, universidades..., es decir, aquellas donde se desarrollen habitualmente las AAE o muy directamente vinculadas a ellas.

En este sentido puntualizaremos que no se está teniendo en cuenta en este análisis las AAE que se desarrollan como uso compatible con el uso residencial. Dado que este estudio se desarrolla a nivel regional y estas áreas, de características residenciales, suelen ser de tamaño reducido a excepción de algún área de ciudad/municipio de gran tamaño.

Con esta información, obtenemos:

- La cobertura real del suelo. No es extraño que la calificación inicial del suelo no se corresponda con el uso final de este. Por ejemplo, es frecuente, que suelos calificados de industriales en la realidad tengan un uso terciario.

-El grado de consolidación del suelo, es decir, obtendremos la parte que se ha desarrollado
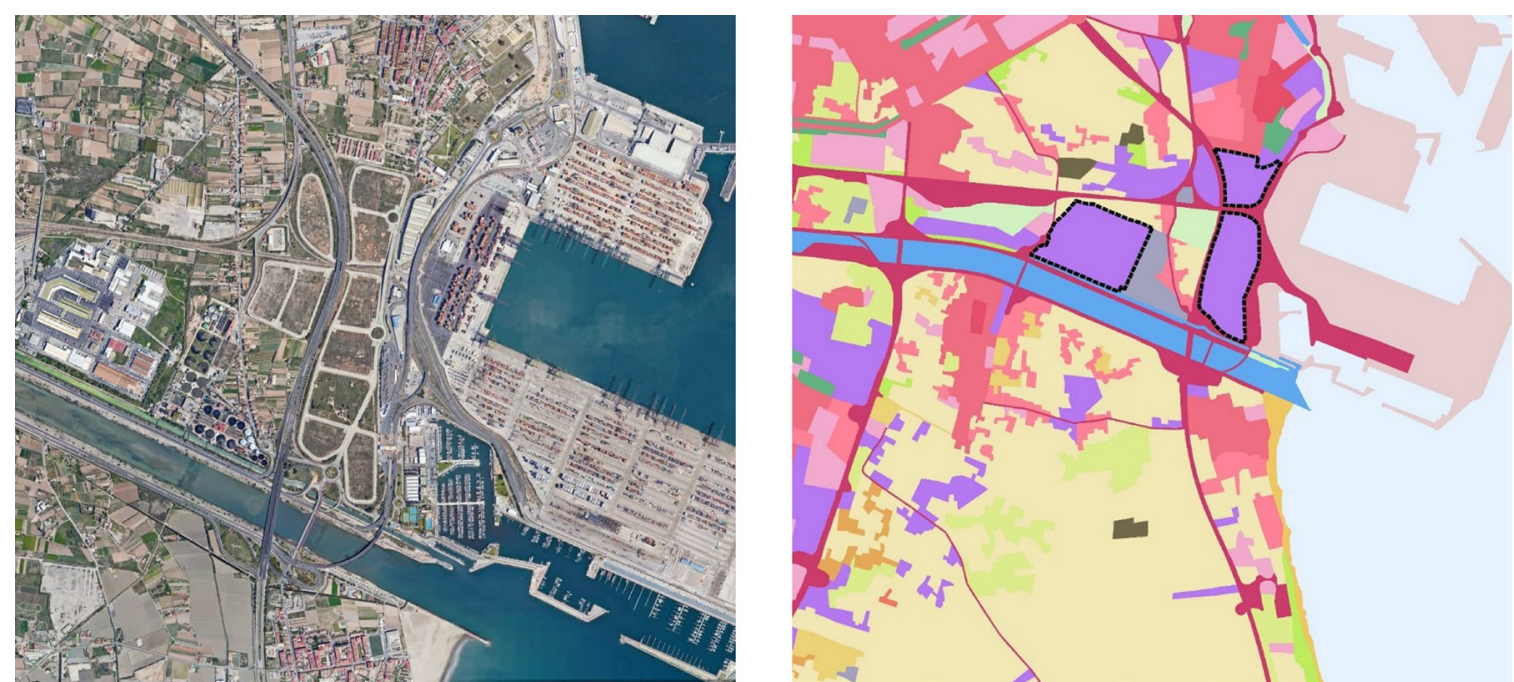

Fig. 2. La cobertura de la ZAL (en morado) se corresponde con "industrial", en la ortofoto vemos que las parcelas no están edificadas. En su lado oeste vemos otra zona industrial que sí se encuentra consolidada por la edificación. Ortofoto 2018 (Fuente: Google Earth) y coberturas del SIOSE 2014 (Fuente: Instituto Geográfico Nacional) 
efectivamente. Pero este sistema no diferencia si el suelo se encuentra consolidado por la edificación o simplemente, está urbanizado. A modo de ejemplo reproducimos la zona de actividades logísticas (ZAL) del Puerto de Valencia (Fig. 2)

Por tanto, necesitaremos completar esta información, con sistemas que nos ofrezcan la presencia de edificaciones en las áreas de estudio. Para ello, podemos contar con la siguiente información:

Cartografía Oficial de la Comunitat Valenciana

El Institut Cartogràfic Valencià ofrece la serie cartográfica vectorial de referencia a escala 1:5000 en 3D de la Comunitat Valenciana. La última versión se realizó mediante restitución fotogramétrica a partir de vuelos digitales comprendidos entre los años 2005-2010, con las siguientes características:

El sistema geodésico de Referencia, ETRS89, proyección UTM en el huso 30. Datum vertical: altitudes referidas al nivel medio del Mar Mediterráneo en Alicante. Código EPSG 25830. Precisión Planimétrica 1 metro. Precisión Altimétrica 1,25 metros. En estas restituciones podemos localizar las construcciones presentes en el territorio.

\section{Catastro}

Aunque no es la finalidad de esta cartografía, con ella, podemos obtener muchos de los datos que con las anteriores no teníamos. Además de localizar las construcciones existentes en el territorio, obtenemos una serie de datos muy útiles para el trabajo que nos ocupa: número de alturas, uso de la edificación, distribución de parcelas catastrales (y, por tanto, de la propiedad), edificabilidad construida, etc.

\section{Infraestructura Verde}

El desarrollo de un territorio no puede ser ajeno al valor intrínseco del mismo, así, se define, la infraestructura verde como "un sistema territorial que incluye los espacios con valores ambientales, paisajísticos y patrimoniales, así como sus conexiones, que resulten necesarios para mantener los procesos ecológicos básicos del territorio". La infraestructura verde es un concepto que debe definirse a distintas escalas territoriales, como paso previo a cualquier actuación urbanística y su grado de protección se adaptará a la prescrita por las distintas legislaciones que se apliquen a cada espacio. Es numerosa la casuística de espacios que la conforman, la Ley Urbanística Valenciana los enumera así:

-Los espacios que constituyen la Red Natura 2000 (Ley 42/2007, de 13 de diciembre, del Patrimonio Natural y Biodiversidad).

-Los espacios naturales (Ley 11/1994, de 27 de diciembre, de Espacios Naturales Protegidos de la Comunitat Valenciana, incluyendo, en su caso, sus áreas de amortiguación).

-Las áreas protegidas formalmente designadas por instrumento Internacionales (Ley 42/2007, de 13 de diciembre, del Patrimonio Natural y la Biodiversidad)

-Las zonas húmedas y las cavidades subterráneas catalogadas (Ley 11/1994, de 27 de diciembre, de Espacios Naturales Protegidos de la Comunitat Valenciana)

-Los montes de dominio público y de utilidad pública o protectores catalogados.

-Los suelos forestales estratégicos (Plan General de Ordenación Forestal de la Comunitat Valenciana formulado en desarrollo de la Ley 3/1993, Forestal de la Comunitat Valenciana)

-Los espacios litorales de interés ambiental $\mathrm{y}$ cultural no incluidos en los supuestos anteriores.

-Los espacios de interés cultural (artículo 21.3, de la Ley de Ordenación del Territorio y Protección del Paisaje, según redacción dada por la Ley 12/2009, de 23 de diciembre, de Medidas Fiscales, de Gestión Administrativa y Financiera, y de Organización de la Generalitat) -Las zonas que se encuentren sometidas a riesgo de inundación, de acuerdo con lo establecido al respecto en el Plan de Acción Territorial de carácter sectorial sobre Prevención del Riesgo de Inundación en la Comunitat Valenciana y sus modificaciones y revisiones.

-Las zonas que presenten un riesgo significativo de erosión o contaminación de acuíferos, definidas y delimitadas de acuerdo con los criterios y normas establecidas en la Ley de Ordenación del Territorio y Protección 
del Paisaje y, en su caso, por un Plan de Acción Territorial confeccionado a tal efecto.

-Las zonas críticas respecto a cualquier riesgo natural e inducido significativo, definidas y delimitadas de acuerdo con los criterios y normas establecidas en la Ley de Ordenación del Territorio y Protección del Paisaje y, en su caso, por un Plan de Acción Territorial.

-Las áreas en las que el planeamiento territorial, ambiental y urbanístico, se establezca explícitamente como adecuadas para su incorporación a la infraestructura verde

-Los espacios de la zona marina, que reúnan valores merecedores de su inclusión.

-Las áreas, espacios y elementos que garanticen la adecuada conectividad territorial y funcional entre los diferentes elementos constitutivos de la infraestructura verde, con especial referencia a las áreas fluviales y los conectores ecológicos y territoriales.

La Infraestructura Verde a escala regional es facilitada por el IDEV, a continuación, se muestra un zoom de la misma en la provincia de Valencia (Fig. 3).

\section{Afecciones físicas y jurídicas}

En la planificación urbanística y territorial, por su carácter interdisciplinar, convergen múltiples intereses que afectan a los diferentes órganos de las Administraciones Públicas. Se deben recoger aquellos elementos existentes en el territorio que, consecuencia de su propia legislación sectorial, generan una serie de afecciones que deben tenerse en cuenta al acometer cualquier actuación. Algunas de estos espacios "afectos" se encuentran recogidos en la propia infraestructura verde, tal es el caso del ancho legal de las vías pecuarias. Otros, sólo lo están en parte, y otros no lo están, por lo que deberán conocerse y conocer la normativa que les rige. Las afecciones más importantes para el tema que nos ocupa, y que no siempre se encuentran incluidas en la infraestructura verde, son las derivadas de las infraestructuras territoriales, tales como carreteras, ferrocarriles, aeropuertos y redes de transporte de energía eléctrica. Otras afecciones a conocer son las derivadas de la legislación en materia de Costas, Forestal, Hidráulica (cauces) y Patrimonio Histórico. En la actualidad la cartografía de estas afecciones no se encuentra a disposición del público en la Comunitat Valenciana, por lo que suele elaborarse manualmente.

\section{Análisis inicial en la Comunitat Valenciana}

La Comunitat Valenciana tiene una realidad política y social que indica altos valores medioambientales y paisajísticos y diferencias considerables en la densidad de población desde la costa hacia el interior, que coincide con las zonas más abruptas del territorio. Las

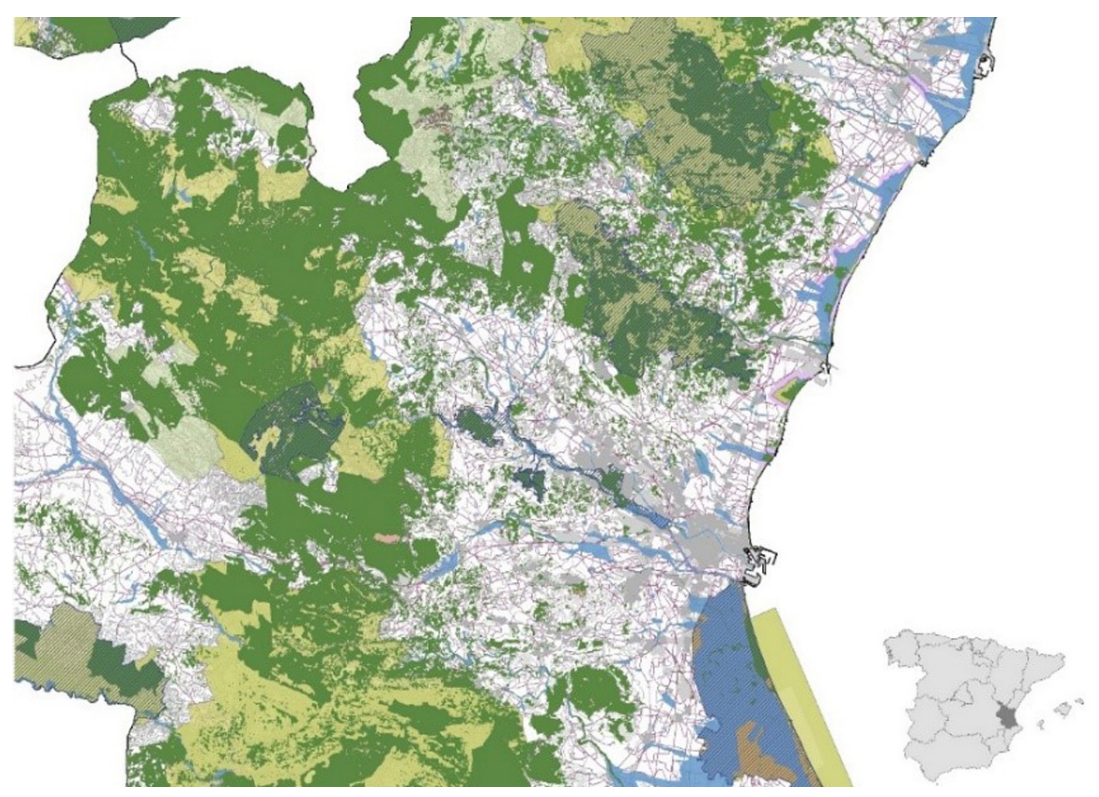

Fig. 3. Infraestructura Verde a nivel regional en la provincia de Valencia. Fuente: Elaboración propia a partir de datos de la IDEV. 


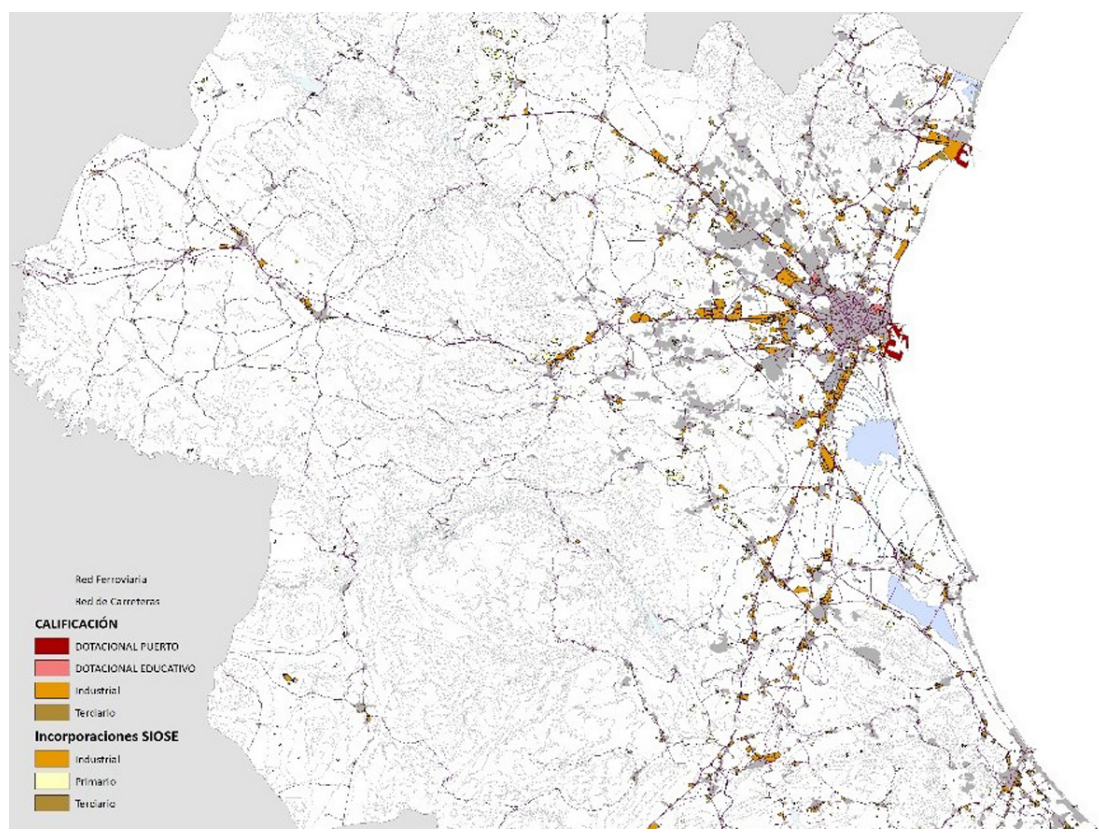

Fig. 4. Localización de los suelos destinados a AAE en la provincia de Valencia. Fuente: Elaboración propia a partir de la cartografía del IDEV.
AAE se concentran fuertemente en las zonas cercanas a la costa, y lógicamente entorno a las principales vías de comunicación. (Fig. 4).

Se observa la gran relación entre las principales infraestructuras de transporte $\mathrm{y}$ la localización de las AAE. Con estos datos obtenidos resulta sencillo obtener variables que nos facilitan una jerarquización de las áreas, y nos indican algunos criterios de actuación. Los principales nodos logísticos de la Comunitat Valenciana han sido analizados en el Estudio del Sector Logístico de la Comunitat Valenciana de noviembre de 2018 y coinciden con las grandes concentraciones industriales. (Fig. 5)

Siempre con la base de la infraestructura verde del territorio ( $\mathrm{y}$ afecciones), que son el verdadero tapiz territorial en el que debe enmarcarse el desarrollo urbanístico y con el resto de datos que hemos obtenido, se propone buscar el siguiente nivel de la jerarquización de las áreas:

1.-Localizamos las áreas económicas de mayor tamaño existentes en la comarca que es el ámbito de análisis elegido. El tamaño de las áreas económicas es uno de los factores que índica su importancia relativa (Fig. 6)

2.-Analizamos el suelo realmente desarrollado del total planificado. Clasificar y desarrollar suelo en exceso, disminuye el recurso suelo y agrava el proceso de degradación de las AAE existentes.

De los datos del IDEV obtenemos la situación urbanística con la zonificación del suelo y tomamos las calificaciones de terciario e industrial. $\mathrm{Y}$ de los datos del SIOSE, obtenemos las coberturas del suelo, si intersectamos ambas capas obtendremos la parte de los suelos terciarios e industriales con las coberturas apropiadas a estos usos. Y, por tanto, un porcentaje de desarrollo. Como se ha indicado en puntos anteriores, un suelo
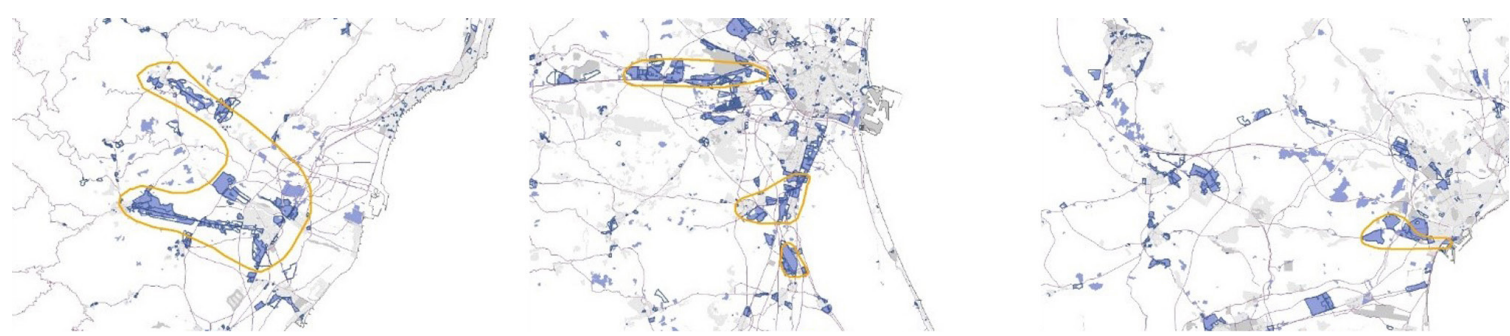

Fig. 5. Estudio del Sector Logístico de la Comunidad Valenciana. Áreas de Castellón, Valencia y Alicante. Noviembre 2018. 

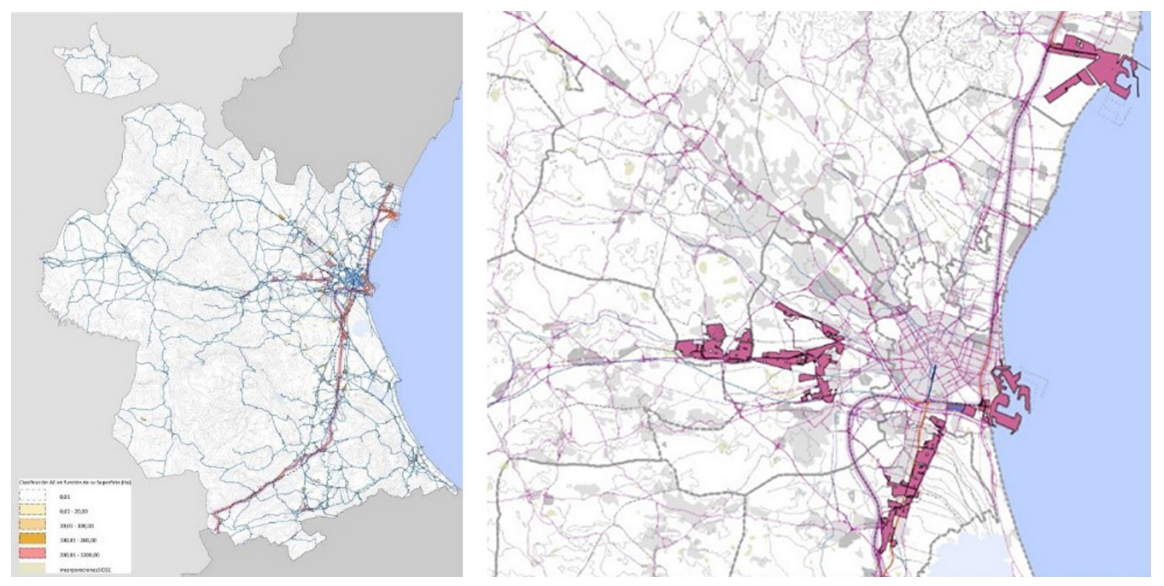

Fig. 6. Clasificación de las AAE en función de su superficie y zoom en las mayores de $500 \mathrm{Ha}$ de superficie. Fuente: Elaboración propia a partir de la cartografía del ICV. desarrollado por el SIOSE es aquél que se encuentra urbanizado. A partir de ahí, puede estar o no consolidado por la edificación. (Fig. 7)

En dicha figura, los contornos azules indican el suelo planificado, y el relleno del mismo color la parte desarrollada. Se observa también zonas desarrolladas que no tienen el contorno que indica la calificación de industrial o terciario. Esto se debe a que las AAE aisladas en el territorio se desarrollan a través de una figura de planeamiento que no está recogida en la base del IDEV. Para observar qué parte de las zonas urbanizadas están consolidadas bastará con superponer la información catastral o de una cartografía detallada. (Fig. 8)

3.-Analizamos también la proximidad a las principales infraestructuras de transporte y a las capitales provinciales y comarcales. Es tremendamente importante el factor localización de las AAE y su conectividad.

De primera magnitud, se considera la conectividad con las infraestructuras de transporte principales, puertos, instalaciones logísticas del ferrocarril y las principales autovías y autopistas. En cuanto a la cercanía a la capital de la comarca, es un factor importante por los servicios que en ellas se concentran y de los que dependen las AAE. Ofrecen otras ventajas como la de la movilidad sostenible, la mezcla de usos y la posibilidad de la integración con el municipio. A través de una operación geométrica que considera una influencia directa una distancia de $1 \mathrm{~km}$, podemos grafiar esta influencia. Genéricamente observamos que hay más AAE donde hay más concentración de áreas de influencia: un $81 \%$ de las AAE están dentro de una o más áreas de influencia. (Fig. 9)

Una vez elegidas las áreas más importantes,

Fig. 7. Planeamiento $y$ desarrollo en las cercanías de Valencia. Fuente: Elaboración propia a partir de datos del IDEV.

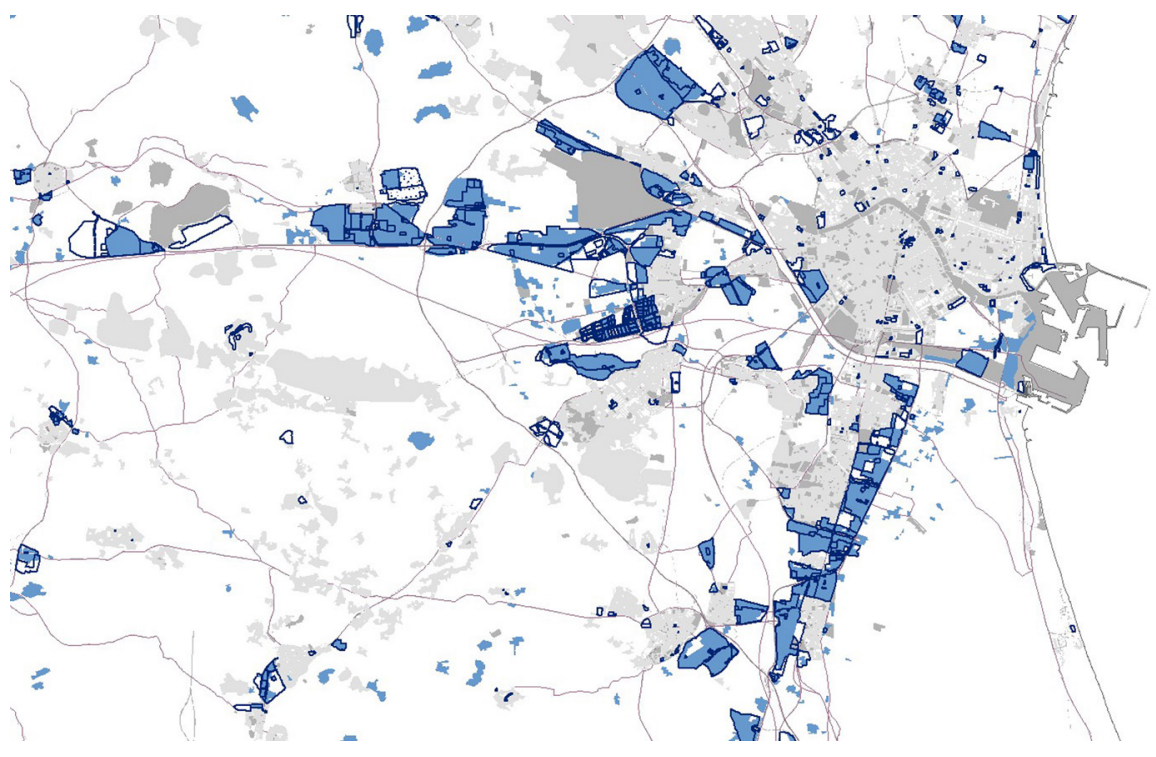


Fig. 8. Planeamiento y desarrollo en las cercanías de Valencia. Detalle del área NW del Área Metropolitana de Valencia. Fuente: Elaboración propia a partir de datos del IDEV.

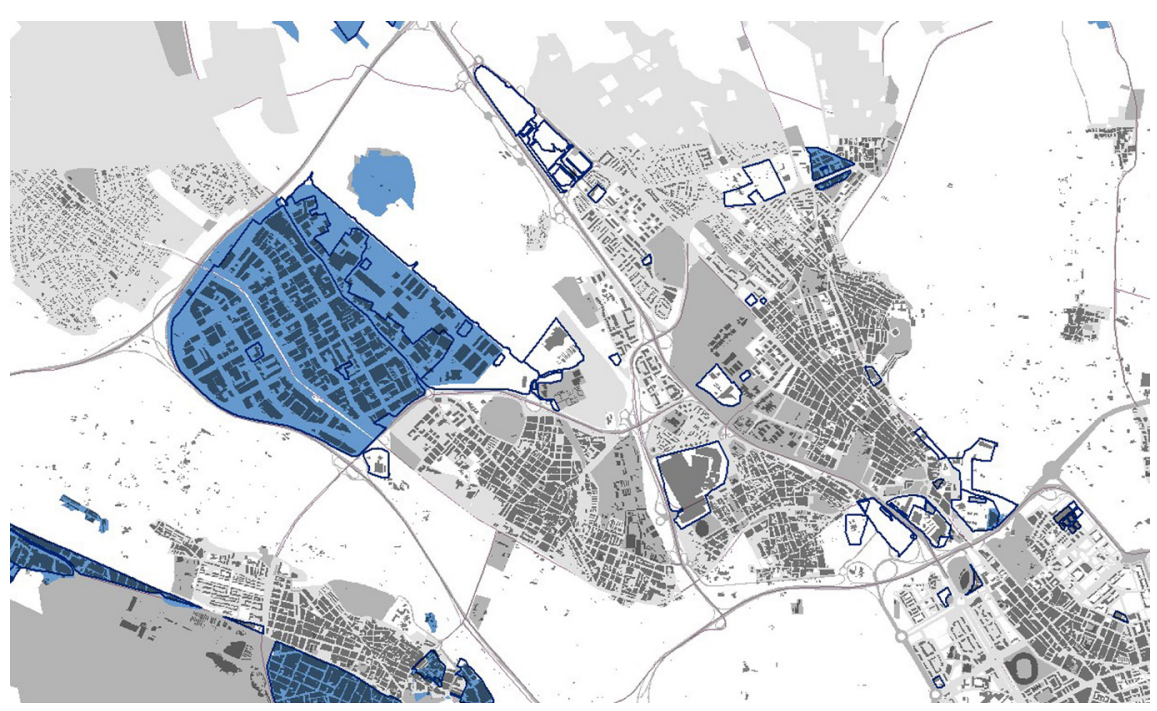

resulta muy sencillo pasar a un análisis más detallado, primero, comprobando que son o pueden ser realmente las áreas líderes del ámbito, analizar a menor escala sus particularidades, valorando sus necesidades particulares y en definitiva, proponiendo unas mejoras y un desarrollo que debe, en aras del desarrollo sostenible, primar la regeneración y el crecimiento interior. (Fig. 10)

\section{Conclusiones}

Del análisis expuesto, podemos decir que, en la Comunitat Valenciana, con sus características particulares, la localización de los suelos de actividad económica se encuentra fuertemente relacionada con las principales infraestructuras de transporte.

La necesaria jerarquización de las áreas para

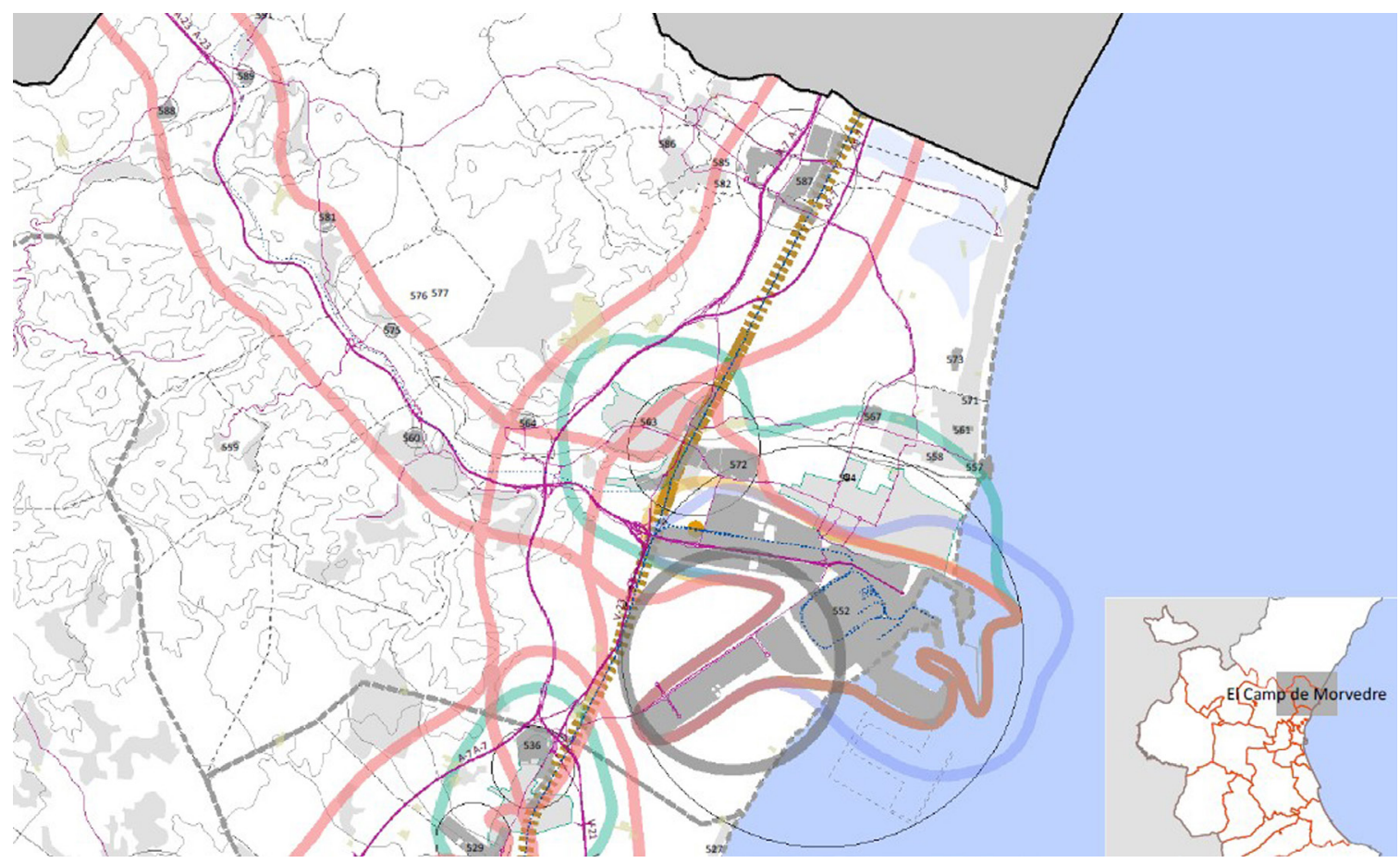

Fig. 9. Proximidad de las AAE a las principales infrestructuras de transporte y a las capitales comarcales. Área del Camp de Morvedre y el núcleo de Sagunt. Fuente: Elaboración propia a partir de datos del IDEV. 


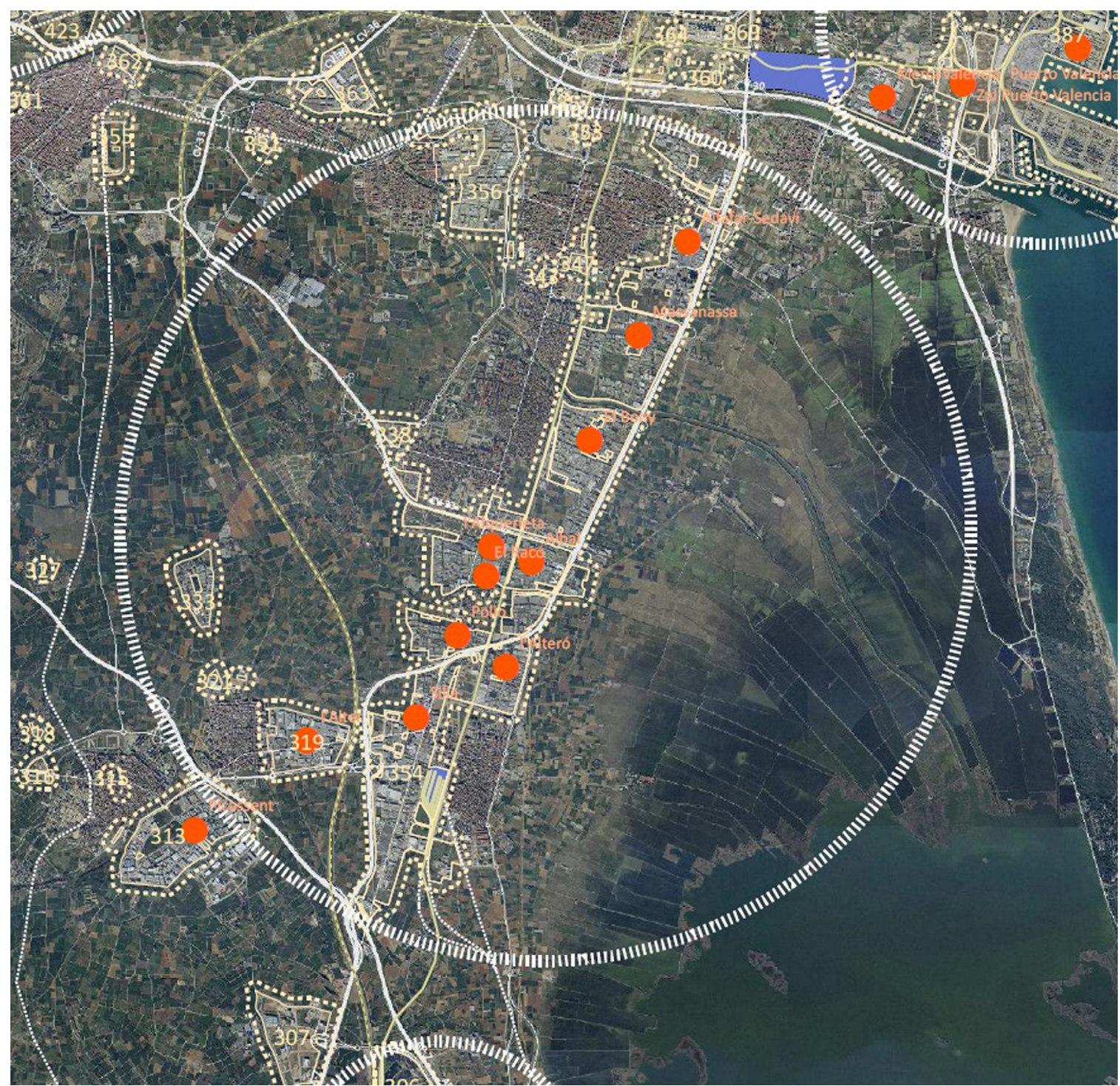

Fig. 10. Análisis detallado de la AAE de la Zona Sur del Área Metropolitana de Valencia. Fuente: Elaboración propia a partir de datos del IDEV.

una adecuada planificación a nivel territorial debe basarse en el tamaño de las mismas, de su estado de desarrollo y de su proximidad a las principales infraestructuras de transporte y a las capitales provinciales y comarcales.

Una vez obtenidos estos grandes factores, y de un análisis más detallado de las mismas podemos decir que:

-Se observa un deterioro de las áreas de suelo dedicadas a actividades económicas, debido a múltiples factores entre los que se encuentran la carencia de una política de apoyo a la rehabilitación, el abandono por obsolescencia técnica, la reubicación por efecto de la globalización y los efectos de la reciente recesión económica.

-La falta de planificación territorial ha provocado una distribución dispersa en el territorio, conurbaciones indeseadas o faltas de conexión entre municipios limítrofes.

-Frente a la fuerte demanda del sector logístico en determinadas áreas estratégicas del territorio, la Comunitat Valenciana carece de suelos de carácter industrial preparados para acoger estos usos, que deberían estar fuertemente vinculados a la presencia del Corredor Ferroviario Mediterráneo en este momento de apoyo institucional para su desarrollo.

-Encontramos una ausencia de la gestión, 
pública o privada de las áreas de actividad económica, que permitiría mejorar el funcionamiento de los equipamientos e instalaciones comunes, desarrollar temas inmobiliarios y de marketing de las áreas o gestionar las sinergias entre ellas, optimizando su funcionamiento.

\section{Referencias}

Martínez Pérez, Francisco Juan, Blasco Sánchez, María Del Carmen, Cano Hurtado, Juan Jaime, Escoms Martínez, Ana Isabel, Noviembre 2016 "El corredor mediterráneo ferroviario como oportunidad. Análisis y perspectivas del suelo destinado a actividades económicas en la provincia de Valencia" DYNA Ingeniería e Industria Volumen 91 Páginas 613 - 618. DOI: 10.6036/7884

Institut Catalá del Sòl. 2007. Sectores de Actividad Económica. Libro de estilo. Barcelona: Institut Catalá del Sòl.

López Groh, Francisco. 2011. La regeneración de áreas industriales. Madrid: Sepes Entidad Estatal de Suelo.

Pellenbarg, Piet. 2000. "Sustainable Business Sites in the Netherlands: A Survey of Policies and Experiences." Journal of Environmental Planning and Management. 45. 10.1080/09640560120100196.

Font Arellano, Antonio, Vecslir Peri, Lorena, Maristany Jackson, Lorena, Mas Artigas, Silvia. 2012. Patrons Urbanístics de les Activitats Econòmiques. Regió Metropolitana de Barcelona. Institut d'Estudis Territorials. ISBN: 978-84-3938874-6. 